\title{
Kidney Injury Molecule-1 Mediated Autophagy Pathway Participates in Ischemic Preconditioning Evoked Renal Protection
}

\section{Subo Qian}

Shanghai Jiaotong University School of Medicine Xinhua Hospital https://orcid.org/0000-0001-60976484

\section{Xinyan Li}

Shanghai Jiao Tong University

\section{Zhengqin Gu}

Shanghai Jiaotong University School of Medicine Xinhua Hospital

Haibo Shen ( $\nabla$ shenhaibo@xinhuamed.com.cn)

https://orcid.org/0000-0002-1183-6570

\section{Research article}

Keywords: ischemic preconditioning (IPC), ischemia-reperfusion injury (IRI), kidney injury molecule-1 (KIM1), major histocompatibility complex class II (MHC II), conditional knockout (cKO)

Posted Date: May 20th, 2020

DOl: https://doi.org/10.21203/rs.3.rs-26737/v1

License: (1) This work is licensed under a Creative Commons Attribution 4.0 International License. Read Full License 


\section{Abstract \\ Background}

Ischemia-reperfusion injury (IRI) is one of the most vital pathogenesis causing kidney injury, especially during the perioperative periods of partial nephrectomy and renal transplantation, but lacking of effective prevention and treatment strategies. This study was conducted to investigate the influences of ischemic preconditioning (IPC) on the pathological process of mouse bilateral IRI, and to figure out the role of kidney injury molecule-1 (KIM-1) mediated autophagy pathway in this process.

\section{Methods}

Nephron major histocompatibility complex class II (MHC II) conditional knockout (cKO) mice (Six2-Cre ${ }^{+/-}$;

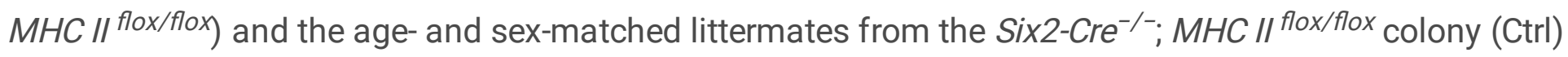
were established to investigate these issues. A 15-minute period of IPC was performed 4 days before the 30-minute of bilateral renal vessel occlusion. Severity of renal IRI in cKO and Ctrl mice with or without IPC were analyzed respectively and correspondingly.

\section{Results}

MHC II cKO mice presented severer kidney injury in both acute and chronic phase of renal IRI. IPC could significantly attenuate ischemia/reperfusion-induced serum creatinine $(\mathrm{sCr})$ and blood urea nitrogen (BUN) increasing, as well as histological KIM-1 expression. However, MHC II cKO mice undergoing IPC showed more deteriorated kidney injury when compared with Ctrl mice, with higher levels of $\mathrm{sCr}, \mathrm{BUN}$, $\mathrm{KIM}-1$ expression in the acute phase, and aggravated interstitial fibrosis in the chronic phase.

\section{Conclusions}

IPC could attenuate renal IRI functionally and histologically. KIM-1 mediated autophagy pathway plays a vital role in the IPC induced renal IRI protection.

\section{Background}

Ischemia-reperfusion injury (IRI) is one of the most vital pathogenesis leading to kidney injury, especially during the perioperative periods of partial nephrectomy and renal transplantation[1]. Multiple strategies, including renoprotective drugs (diuretics, sodium bicarbonate, etc.) and renal replacement therapy have been brought up to prevent or treat renal IRI. However, most of these efforts have yielded limited success[2]. Ischemic preconditioning (IPC) is a kind of endogenous phenomenon that promotes tissue tolerance to IRI by a brief episode of ischemia and subsequent reperfusion before the index ischemic 
event, and may be a highly appealing, nonpharmacological and practical approach to attenuate renal IRI[3].

Since the concept of IPC was firstly proposed by Murry et al. in 1986[4], several studies have assessed the organ protective effect of IPC in various tissues and organs as well as across several species[5-8]. Over the past three decades, both experimental and clinical researches have demonstrated that IPC could enhance kidney tolerance to ischemic injury and has therapeutic potential for the prevention and/or reversal of the pathological sequelae associated with acute kidney injury (AKI)[9-13]. Nitric oxide[14], protein kinase C[15], MAP kinase and MAPKAP kinase 2[16], NF-KB [17], mitochondria[18], microRNA[19], and decreased capacity of immune cells[20], etc. have all been implicated in mediating the protective effects of IPC. But the pivotal mechanisms of IPC still remain unclear.

Kidney injury molecule-1 (KIM-1) mediated epithelial cell phagocytosis of apoptotic or necrotic cells belongs to autophagy pathway which involves the process of KIM-1 mediated phagocytosis to autophagy induction, major histocompatibility complex (MHC) presentation and maintenance of peripheral T-cell tolerance[21], and is reported playing an important role in the process of kidney injury and epithelia renovation in both animal models and human diseases[22]. However, the role of this pathway in IPC evoked renal IRI protection is still unknown.

Thus, this study was conducted to investigate the influences of IPC on the pathological process of mouse bilateral IRI, and to figure out the role of KIM-1 mediated autophagy pathway in this process by establishing a nephron MHC II conditional knockout (cKO) mouse model.

\section{Methods}

\section{Animals}

8-12 week old male nephron MHC II cKO (Six2-Cre ${ }^{+/-} ; \mathrm{MHC} /$ flox/flox $^{\text {f }}$ mice on a C57BL/6J background and the age- and sex-matched littermates from the Six2-Cre ${ }^{-/-} ; \mathrm{MHC} /$ flox/flox colony (used as control (Ctrl)) weighing 20-25 g obtained from animal experiment center (Super-B\&K Laboratory Animal Corp. Ltd, Shanghai, China) were used in all experiments. Mice had free access to food and water, and were housed and maintained at Xinhua Hospital Laboratory Animal Resource Facility. Animals were assessed daily by veterinary staffs at our institution and by qualified investigators in our group. All animal procedures were approved by the Animal Ethics Committee of Xinhua Hospital, and in conformity to the Guide for Care and Use of Laboratory Animals.

\section{Bilateral renal IRI and IPC mouse model}

Briefly, experimental mice were anesthetized with isoflurane (1.6-1.8\%). Under aseptic conditions, two dorsal flank incisions were performed and the renal pedicles of both sides were identified and clamped using a small non-traumatic clamp $\left(4^{\star} 0.75 \mathrm{~mm} / 16 \mathrm{~mm}\right.$, RWD Life Science, San Diego, CA, USA). After visual confirmation of ischemic changes, the kidneys were returned to the retroperitoneal cavity and 
vessel occlusion was maintained for 15 minutes[23]. The clamps were then released and blood flow restoration confirmed visually. Incisions were closed in two layers using 4- 0 absorbable sutures and animals were volume resuscitated using normal saline $(0.2 \mathrm{~mL})$ injected subcutaneously. Subcutaneous buprenorphine $(0.075 \mathrm{mg} / \mathrm{kg})$ was given every $8-12$ hours to ensure post-operative analgesia till 48 hours. 4 days later[23], the same procedure was performed again expect for the vessel occlusion maintaining for 30 minutes. Animals in sham-operated group were anesthetized and two flank incisions performed, renal pedicles dissected but not clamped. They were maintained for corresponding minutes mentioned above and the incisions were then closed in two layers.

\section{Tissue harvest}

To study acute phase of renal IRI, we allowed reperfusion for 2 days post-ischemia. To investigate postischemic progression to fibrosis, mice were followed for 42 days after injury. Following the reperfusion phase, animals were anesthetized, blood was obtained by mice tail at the time-point of 24 hours, 72 hours, and weekly from 1 week to 5 weeks after the surgery, and by cardiac puncture at the time-point of 6 weeks. Native kidneys were obtained from healthy anesthetized animals and processed immediately.

Mice were euthanized by high concentration of isoflurane. Kidneys were surgically removed under anesthesia after reperfusion for $20 \mathrm{~mL}$ of phosphate-buffered saline (PBS), and then sectioned after removal of the renal capsule and extrarenal structures. Each kidney was sectioned along the transverse axis into two pieces: upper (3- to 4-mm length) and lower (3-to 4-mm length). The upper pieces were fixed in $4 \%$ paraformaldehyde in $1 \mathrm{X}$ PBS for 24 hours at room temperature. Following fixation, tissue samples were routinely processed and embedded in paraffin wax (Fisher Scientific, Pittsburgh, PA, USA), and then for H\&E staining, picrosirius red staining and immunofluorescence staining, etc. The medulla of the lower pieces were removed and the cortex were cut into small pieces and then flashed frozen in liquid nitrogen and then stored at $-80{ }^{\circ} \mathrm{C}$ for further protein studies.

\section{Detection of kidney injury related indicators}

Western blot and immunofluorescence staining were used to detect the expression of KIM-1, cytokines including connective tissue growth factor (CTGF), alpha smooth muscle actin (a-SMA), transforming growth factor- $\beta$ (TGF- $\beta$ ), etc. and collagen I and IV. They were all performed according to the protocols provided by the manufacturer (Sigma-Aldrich, St. Louis, MO, USA). The antibodies for western blot were listed as bellows. KIM-1: primary antibody, goat anti-mouse KIM-1 (1:800, R\&D Systems, Minneapolis, MN, USA); secondary antibody, HRP-conjugated donkey anti-goat IgG antibody (1:5000, Jackson ImmunoResearch Laboratories, WestGrove, PA, USA). CTGF: primary antibody, rabbit anti-mouse CTGF (1:1000, GeneTex, Irvine, CA, USA); secondary antibody, HRP-linked goat anti-rabbit IgG antibody (1:2500, Cell Signaling Technology, Danvers, MA, USA). a-SMA: primary antibody, rabbit anti-a SMA antibody (1:500, abcam, Cambridge, MA, USA ); secondary antibody, goat anti-rabbit IgG H\&L (HRP) (1:2500, abcam, Cambridge, MA, USA). TGF- $\beta$, anti-TGF $\beta 1$ antibody produced in rabbit affinity isolated antibody (1:500, Sigma-Aldrich, St. Louis, MO, USA); secondary antibody, goat anti-rabbit IgG H\&L (HRP) (1:2500, abcam, Cambridge, MA, USA). Those for immunofluorescence staining were as followings. KIM-1, goat 
anti-mouse KIM-1 (1:200, R\&D Systems, Minneapolis, MN, USA) and Cy ${ }^{\text {TM } 3}$ AffiniPure Donkey Anti-Goat IgG $(H+L)$ (1:1000, Jackson ImmunoResearch Laboratories, WestGrove, PA, USA). Collagen IV, rabbit anticollagen IV antibody (1:100, abcam, Cambridge, MA, USA) and goat anti-rabbit IgG H\&L (Cy3 $®)$ (1:1000, abcam, Cambridge, MA, USA). Collagen I, rabbit anti-collagen I antibody (1:500, abcam, Cambridge, MA, USA) and goat anti-rabbit IgG H\&L (Cy3 $®)$ (1:1000, abcam, Cambridge, MA, USA).

Picrosirius red staining and H\&E staining, as well as sCr and BUN measurement were also conducted according to the manufacturer's protocols (abcam, Cambridge, MA, USA).

\section{Statistical analysis}

Statistical comparisons were performed using the statistical software GraphPad Prism 5 (GraphPad Software, San Diego, CA, USA). The intergroup differences were tested using the Student's t test or analysis of variance (ANOVA). Statistical significance was defined as a $p<0.05$.

\section{Results}

\section{Phenotype of the nephron MHC II cKO mouse}

To knock out MHC // gene from nephron, we crossed MHC IIf/fl mice (Six2-Cre ${ }^{-/-} ; \mathrm{MHC} / /$ flox/flox) with transgenic mice expressing Six2-Cre. The Six 2 gene has been linked to abnormalities of craniofacial and kidney development[24]. The findings of our pilot experiments have confirmed that homozygous of Six2$\mathrm{Cre}\left(\mathrm{Six} 2-\mathrm{Cre}^{+/+}\right)$is embryolethal. Consequently, heterozygotes (Six2-Cre ${ }^{+/-}$) were screened to express Cre recombinase in this study. To verify the efficiency of MHC II knockout, cortical primary proximal tubular cells (PTCs) were isolated from Ctrl and cKO mice respectively, and then cultured in Dulbecco's Modified Eagle Medium/Nutrient Mixture F-12 (DMEM/F-12) for 3 days before harvest. Isolated PTCs were used for western blot. As indicated in Fig. 1A, cKO mice were verified prominently lower MHC II expression.

Unless otherwise indicated, we confirmed our findings in both strains (Six2-Cre ${ }^{+/-} ; \mathrm{MHC} /$ flox/flox $^{\text {mice }}$ and Six2-Cre ${ }^{-/-} ;$MHC II flox/flox mice). As showed in Fig. 1B-1G, cKO and Ctrl mice had similar appearance, behavior, body weight, kidney weight and baseline renal function ( $\mathrm{SCr}$ and $\mathrm{BUN})$. Intriguingly, cKO mice presented severer underlying kidney fibrosis (Fig. 1H), implying that MHC II cKO mice were more susceptible to factors inducing kidney injury in the environment, and furthermore affirmed that KIM-1 mediated autophagy pathway played an essential role in physiological kidney injury and epithelia renovation.

\section{KIM-1 mediated autophagy pathway was a protective factor of renal IRI}

Bilateral 30 minutes of ischemia was formulated to establish the severe renal IRI model. The ideograph was shown in Fig. 2A. The time-point of day 2 post-ischemia was chosen to evaluate the acute phase and that of day 42 was optimized to investigate the chronic phase according to our research protocol. cKO 
mice showed dramatical differences in acute functional recovery from IRI, as indicated by diverse recovery patterns of both $\mathrm{sCr}$ and BUN, as well as different PTCs KIM-1 expressions 48 hours after kidney ischemia. However, from 1 week after the index ischemic injury, there were similar patterns of both sCr and BUN variations (Fig. 2B-2E). As for the chronic phase, nephron MHC II cKO led to deteriorated interstitial fibrosis, which was indicated by the results of picrosirius red staining (Fig. 2F). Summing up the above, all these results confirmed that KIM-1 mediated autophagy pathway played a protective role in the pathological process of renal IRI.

\section{IPC attenuated renal IRI functionally and histologically}

The delayed kidney ischemic preconditioning (DIPC) strategy was performed as shown in Fig. 3A. As expected, DIPC significantly attenuated ischemia/reperfusion-induced $\mathrm{sCr}$ and BUN increasing and KIM-1 expression in the early phase when compared to those without DIPC. Furthermore, in the groups of undergoing DIPC, CKO mice showed deteriorated kidney injury when compared to corresponding Ctrl mice. Nevertheless, consistent with the results above, from 1 week after the index ischemic injury, there were similar patterns of both $\mathrm{sCr}$ and BUN variations (Fig. 3B-3E). 48 hours after the index ischemia, renal protein levels of the profibrotic and fibrotic markers were detected by western blot. As shown in Fig. 3F, aSMA, CTGF and TGF- $\beta$, etc. all presented a marked increase in the cKO mice.

In the chronic phase of renal IRI, DIPC also played a protective role. cKO mice suffered from an extremely serious atrophic kidney manifesting the lowest left kidney weight / body weight (Fig. 4A). Consistent with this, H\&E staining of the kidney tissues showed that cKO mice exhibited severer histological kidney injury, as indicated by tubular dilation and distal tubular protein casts, nephrons loss, immunocytes infiltration and deposition of extracellular matrix, and these parameters were all diminished in the kidneys of Ctrl mice (Fig. 4B). Meanwhile, MHC II CKO led to increased renal interstitial fibrosis which was confirmed by picrosirius red staining and immunofluorescence staining of collagens I and IV (Fig. 4C-4E).

In a nutshell, these results implied that DIPC played a protection role in the pathological process of renal IRI, in both acute and chronic phase. Besides, KIM-1 mediated autophagy pathway played a vital role in the IPC induced renal IRI protection.

\section{Discussion}

AKI is a common critical illness with high risks of morbidity and mortality. Around $50 \%$ will eventually develop to chronic kidney disease, and $8.1 \%$ of these patients will progress to end-stage renal disease and require dialysis or renal transplantation[25,26]. Plenty of pathogenesis etiologies have been reported causing AKI, and IRI is one of the most important. Previous studies by Brooks et al. have discovered an important role of KIM-1 mediated autophagy pathway in recovery from renal IRI. As reported, KIM-1 mediated autophagy pathway involves the process of KIM-1 mediated phagocytosis to autophagy induction, MHC presentation and maintenance of peripheral T-cell tolerance, and is a protective factor in the process of kidney injury and epithelia renovation by regulating the PTCs immune reaction[21]. In this 
study, the phenomenon that KIM-1 mediated phagocytosis played a protective role in the pathological process of renal IRI was verified again in mouse bilateral IRI model by the construction of nephron MHC II cKO technique. Meanwhile, KIM-1 mediated autophagy pathway also participated in the IPC induced renal IRI protection. This is supported by Zarbock et al.'s hypothesis that renoprotection is mediated mainly through release of damage-associated molecular patterns that interact with pattern recognition receptors on renal tubular epithelial cells [12].

Although the evidences that IPC could attenuate kidney injury in several species and even in humans are irrefutable, IPC is still not currently recommended for renal transplantation or partial nephrectomy in clinical settings. The primary reason is that though numerous studies have investigated IPC and its effects, there has been no established or verified standard protocol of how to conduct the procedure, which may be attributable to the wide variety of methods that used for various facets of IPC, including the time window of ischemia[3]. Time window is the time between IPC and the index ischemic event. According to the time window of ischemia, IPC has been divided into acute IPC (AIPC, occurring immediately and lasting for 1-2 hours) and delayed IPC (DIPC, occurring 24-48 hours after the IPC stimulus and lasting for several days even weeks), which are both reported protective $[27,28]$. Thought it is generally believed that DIPC is more effective than AIPC in reducing renal IRI[13], there is still no specific time window of ischemia has been recommended to maximize the renal protective effect of IPC. Zhang et al. previously studied and demonstrated that IPC could reduce renal IRI when conducted 4 days before the index ischemia [23]. Thus, we chose the time window of 4 days in this research, and confirmed that DIPC could significantly attenuate renal IRI. However, whether is this the most ideal time window still needs more evidences and to be verified in other animal models or even in clinical settings. Secondly, it might be detrimental to apply local IPC to humans by repeatedly clamping and declamping renal vessels. As a result, some studies have studied the effect of remote IPC (RIPC), triggered by brief episodes of ischemia and reperfusion applied in distant tissues or organs before injury of the target organ[12]. Recently, experimental and clinical evidences have demonstrated that RIPC might be an effective, noninvasive and inexpensive strategy to protect kidneys from injury[12]. More intriguingly, no difference in efficacy between local and remote IPC has been observed. Nevertheless, a therapeutic index for RIPC and the appropriate intensity of RIPC have not been established, which limits its clinical application. Thirdly, the renal protective effects of IPC in renal transplantation or partial nephrectomy in clinical settings are still ambiguous. Huang et al. investigated the effect of IPC on renal function in patients undergoing laparoscopic partial nephrectomy, and found that IPC was associated with a lower incidence of glomerular filtration rate (GFR) reduction (measured by renal scintigraphy) at 1 month after surgery (8.8\% versus $15 \%, p=0.03$ ). However, there were no differences in sCr level or estimated GFR (eGFR) at 1 and 6 months between IPC group and control group[29]. Besides, as reported by Chen at el., IPC did not improve early renal function in patients receiving living-donor renal transplantation[30]. Thus, the clinical application of IPC in patients undergoing partial nephrectomy or renal transplantation still needs more research supports. Further large multicenter trials are required to establish the clinical benefits of IPC as well as to understand the optimal dose and patient selection. 


\section{Conclusions}

The present study determines that IPC plays a protective role in the pathological process of renal IRI. Selective deletion of nephron MHC II deteriorates kidney injury and tubulointerstitial fibrosis resulting from renal IRI. KIM-1 mediated autophagy pathway plays a vital role in IPC evoked renal IRI protection. And clearly, more researches are desiderated to form a better evidentiary basis for the use of IPC, to understand the therapeutic potential and potential risks, to determine when and in whom the intervention works.

\section{Abbreviations \& Acronyms}

AIPC, acute ischemic preconditioning

AKI, acute kidney injury

ANOVA, analysis of variance

a-SMA, alpha smooth muscle actin

BUN, blood urea nitrogen

cKO, conditional knockout

CTGF, connective tissue growth factor

Ctrl, control

DIPC, delayed ischemic preconditioning

eGFR, estimated glomerular filtration rate

GFR, glomerular filtration rate

IPC, ischemic preconditioning

$\mathrm{IRI}$, ischemia-reperfusion injury

KIM-1, kidney injury molecule 1

MHC, major histocompatibility complex

PBS, phosphate-buffered saline

PTC, proximal tubular cell

RIPC, remote ischemic preconditioning 
$\mathrm{sCr}$, serum creatinine

TGF- $\beta$, transforming growth factor- $\beta$

\section{Declarations}

\section{Consent to participate}

Not applicable.

\section{Ethical approval}

All animal procedures were approved by the Animal Ethics Committee of Xinhua Hospital, and in conformity to the Guide for Care and Use of Laboratory Animals.

\section{Consent for publication}

Not applicable.

\section{Availability of data and material}

The datasets generated and analyzed during the current study are available from the corresponding authors, Haibo Shen and Zhengqin Gu, upon reasonable request.

\section{Conflict of Interest}

The authors declare that they have no conflict of interest.

\section{Funding}

Not applicable.

\section{Authors' contributions}

SB Qian: study design, perform the experiments, data analysis, manuscript writing and editing

XY Li: study design, perform the experiments, data analysis, manuscript editing

YJ Yu: help to perform the experiments, manuscript editing 
ZQ Gu: study design, data analysis, manuscript editing, supervision

HB Shen: study design, data analysis, manuscript writing and editing, supervision

All authors have read and approved the manuscript.

\section{Acknowledgements}

The authors thank Craig R. Brooks (Vanderbilt University Medical Center) for his proposal of the research idea and technical assistance.

\section{References}

1. Menting TP, Wever KE, Ozdemir-van Brunschot DM, Van der Vliet DJ, Rovers MM, et al. Ischaemic preconditioning for the reduction of renal ischaemia reperfusion injury. Cochrane Database Syst Rev. 2017;3:CD010777.

2. Hu J, Liu S, Jia P, Xu X, Song N, et al. Protection of remote ischemic preconditioning against acute kidney injury: a systematic review and meta-analysis. Crit Care. 2016;20:111.

3. Yoon YE, Lee KS, Choi KH, Kim KH, Yang SC, et al. Preconditioning strategies for kidney ischemia reperfusion injury: implications of the "time-window" in remote ischemic preconditioning. PLoS One. 2015;10:e0124130.

4. Murry CE, Jennings RB, Reimer KA. Preconditioning with ischemia: a delay of lethal cell injury in ischemic myocardium. Circulation. 1986;74:1124-36.

5. Perez-Pinzon MA, Xu GP, Dietrich WD, Rosenthal M, Sick TJ. Rapid preconditioning protects rats against ischemic neuronal damage after 3 but not 7 days of reperfusion following global cerebral ischemia. J Cereb Blood Flow Metab. 1997;17:175-82.

6. Hotter G, Closa D, Prados M, Fernandez-Cruz L, Prats N, et al. Intestinal preconditioning is mediated by a transient increase in nitric oxide. Biochem Biophys Res Commun. 1996;222:27-32.

7. Yin DP, Sankary HN, Chong AS, Ma LL, Shen J, et al. Protective effect of ischemic preconditioning on liver preservation-reperfusion injury in rats. Transplantation. 1998;66:152-7.

8. Jerome SN, Akimitsu T, Gute DC, Korthuis RJ. Ischemic preconditioning attenuates capillary no-reflow induced by prolonged ischemia and reperfusion. Am J Physiol. 1995;268:H2063-7.

9. Kapitsinou PP, Haase VH. Molecular mechanisms of ischemic preconditioning in the kidney. Am J Physiol Renal Physiol. 2015;309:F821-34.

10. Park KM, Chen A, Bonventre JV. Prevention of kidney ischemia/reperfusion-induced functional injury and JNK, p38, and MAPK kinase activation by remote ischemic pretreatment. J Biol Chem. 2001;276:11870-6.

11. Zarbock A, Schmidt C, Van Aken H, Wempe C, Martens S, et al. Effect of remote ischemic preconditioning on kidney injury among high-risk patients undergoing cardiac surgery: a randomized 
clinical trial. JAMA. 2015;313:2133-41.

12. Zarbock A, Kellum JA. Remote Ischemic Preconditioning and Protection of the Kidney-A Novel Therapeutic Option. Crit Care Med. 2016;44:607-16.

13. Wever KE, Menting TP, Rovers M, van der Vliet JA, Rongen GA, et al. Ischemic preconditioning in the animal kidney, a systematic review and meta-analysis. PLoS One. 2012;7:e32296.

14. Park KM, Byun JY, Kramers C, Kim Jl, Huang PL, et al. Inducible nitric-oxide synthase is an important contributor to prolonged protective effects of ischemic preconditioning in the mouse kidney. J Biol Chem. 2003;278:27256-66.

15. Speechly-Dick ME, Mocanu MM, Yellon DM. Protein kinase C. Its role in ischemic preconditioning in the rat. Circ Res. 1994;75:586-90.

16. Maulik N, Watanabe M, Zu YL, Huang CK, Cordis GA, et al. Ischemic preconditioning triggers the activation of MAP kinases and MAPKAP kinase 2 in rat hearts. FEBS Lett. 1996;396:233-7.

17. Jiang SH, Liu CF, Zhang XL, Xu XH, Zou JZ, et al. Renal protection by delayed ischaemic preconditioning is associated with inhibition of the inflammatory response and NF-kappaB activation. Cell Biochem Funct. 2007;25:335-43.

18. Halestrap AP, Clarke SJ, Khaliulin I. The role of mitochondria in protection of the heart by preconditioning. Biochim Biophys Acta. 2007;1767:1007-31.

19. Xu X, Kriegel AJ, Liu Y, Usa K, Mladinov D, et al. Delayed ischemic preconditioning contributes to renal protection by upregulation of miR-21. Kidney Int. 2012;82:1167-75.

20. Burne-Taney MJ, Liu M, Baldwin WM, Racusen L, Rabb H. Decreased capacity of immune cells to cause tissue injury mediates kidney ischemic preconditioning. J Immunol. 2006;176:7015-20.

21. Brooks CR, Bonventre JV. KIM-1/TIM-1 in proximal tubular cell immune response. Oncotarget. 2015;6:44059-60.

22. Brooks CR, Yeung MY, Brooks YS, Chen H, Ichimura T, et al. KIM-1-/TIM-1-mediated phagocytosis links ATG5-/ULK1-dependent clearance of apoptotic cells to antigen presentation. EMBO J. 2015;34:2441-64.

23. Zhang T, Song N, Fang Y, Teng J, Xu X, et al. Delayed Ischemic Preconditioning Attenuated Renal Ischemia-Reperfusion Injury by Inhibiting Dendritic Cell Maturation. Cell Physiol Biochem. 2018;46:1807-20.

24. Okello DO, lyyanar PPR, Kulyk WM, Smith TM, Lozanoff S, et al. Six2 Plays an Intrinsic Role in Regulating Proliferation of Mesenchymal Cells in the Developing Palate. Front Physiol. 2017;8:955.

25. Luo F, Shi J, Shi Q, Xu X, Xia Y, et al. Mitogen-Activated Protein Kinases and Hypoxic/Ischemic Nephropathy. Cell Physiol Biochem. 2016;39:1051-67.

26. Mehta RL, Cerda J, Burdmann EA, Tonelli M, Garcia-Garcia G, et al. International Society of Nephrology's Oby25 initiative for acute kidney injury (zero preventable deaths by 2025): a human rights case for nephrology. Lancet. 2015;385:2616-43. 
27. Hausenloy DJ, Yellon DM. The second window of preconditioning (SWOP) where are we now? Cardiovasc Drugs Ther. 2010;24:235-54.

28. Mayer B, Soppert J, Kraemer S, Schemmel S, Beckers C, et al. (2016) Argon Induces Protective Effects in Cardiomyocytes during the Second Window of Preconditioning. Int J Mol Sci 17.

29. Huang J, Chen Y, Dong B, Kong W, Zhang J, et al. Effect of remote ischaemic preconditioning on renal protection in patients undergoing laparoscopic partial nephrectomy: a 'blinded' randomised controlled trial. BJU Int. 2013;112:74-80.

30. Chen $Y$, Zheng $H$, Wang $X$, Zhou Z, Luo A, et al. Remote ischemic preconditioning fails to improve early renal function of patients undergoing living-donor renal transplantation: a randomized controlled trial. Transplantation. 2013;95:e4-6.

\section{Figures}

A
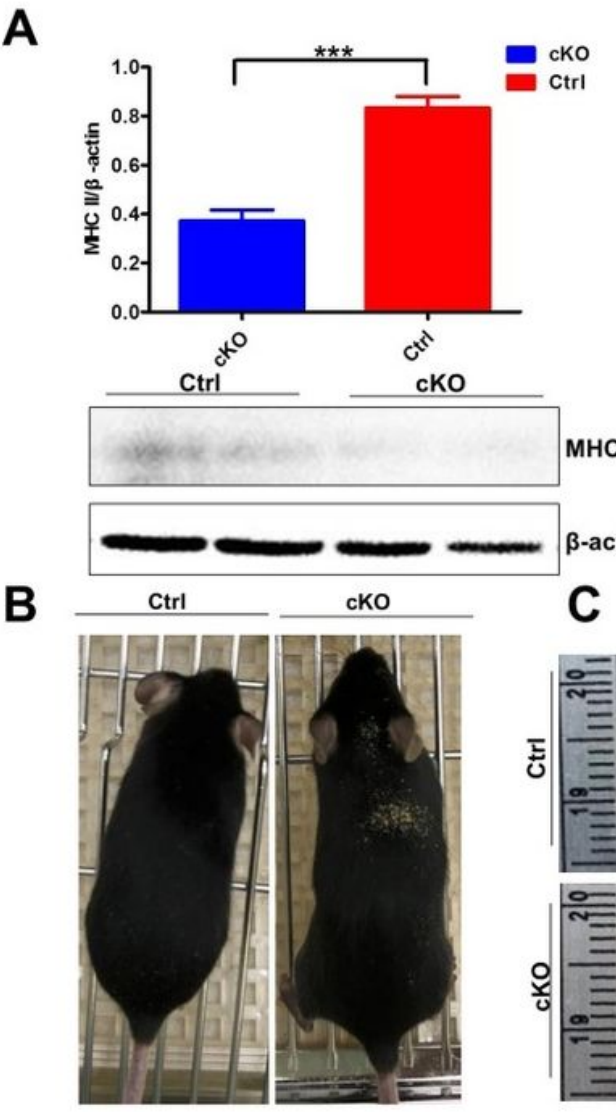

D
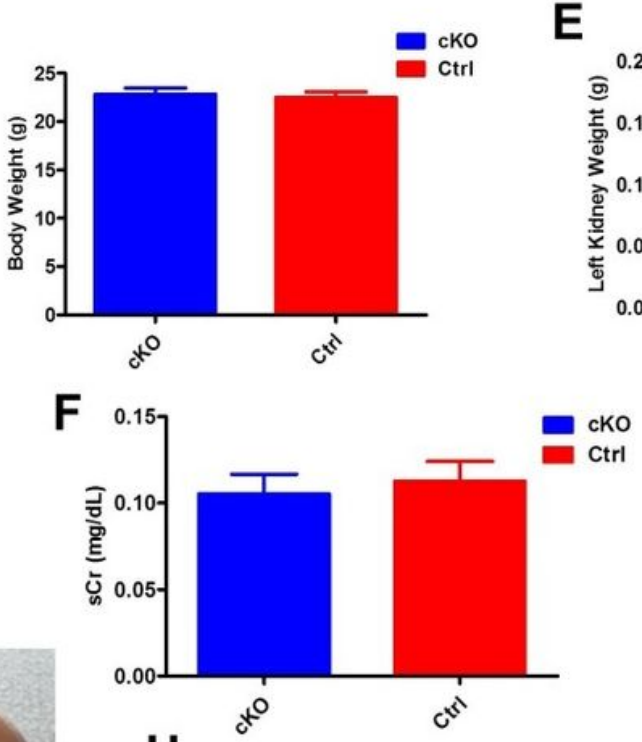

H

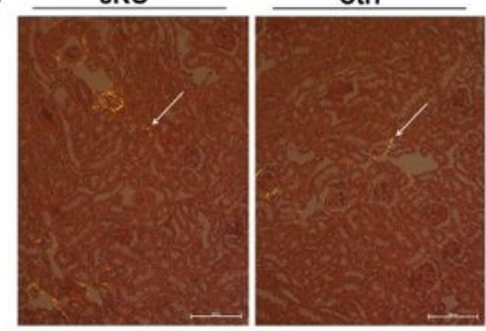

E
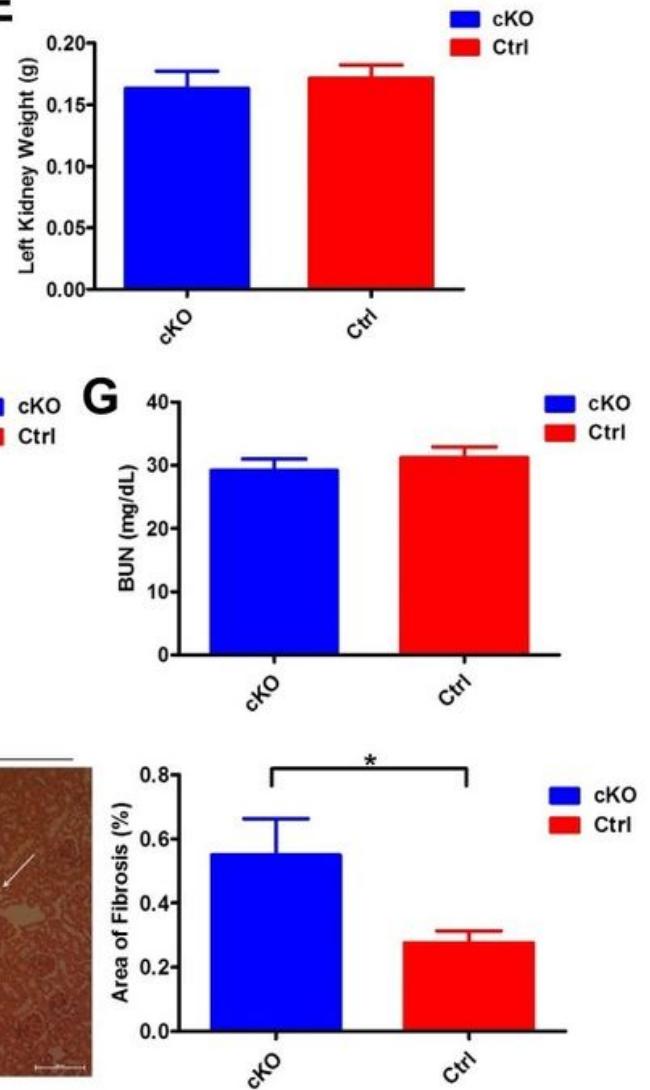

Figure 1

Phenotype of the nephron MHC II cKO mice (A) MHC II expression in primary cultured PTCs. (B-H) Appearance, kidney size, body weight (g), left kidney weight (g), baseline $\mathrm{s} C \mathrm{r}(\mathrm{mg} / \mathrm{dL})$, baseline BUN $(\mathrm{md} / \mathrm{dL})$ and baseline tubulointerstitial fibrosis of $\mathrm{cKO}$ and Ctrl mice. Abbreviations BUN blood urea nitrogen, cKO conditional knockout, Ctrl control, MHC major histocompatibility complex, PTCs proximal 
tubular cells, sCr serum creatinine * meant $p<0.05, * \star \star$ meant $p<0.001$, were assumed as statistically significant. Scale bar: $100 \mathrm{~lm}(\mathrm{H})$
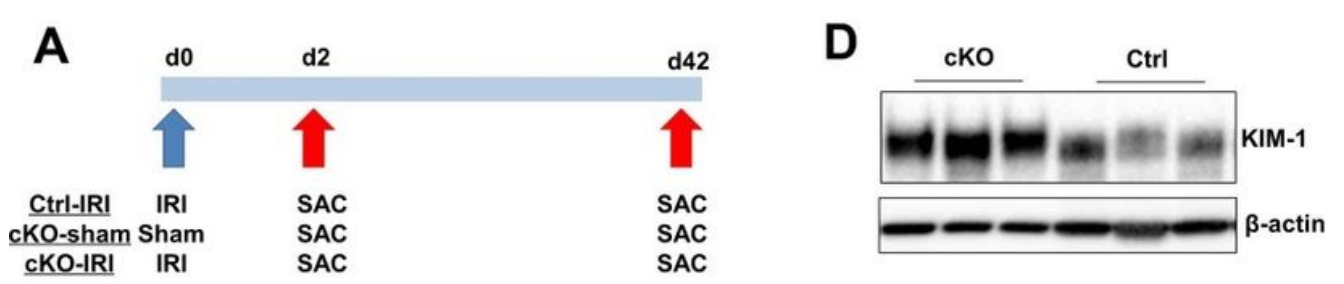

B

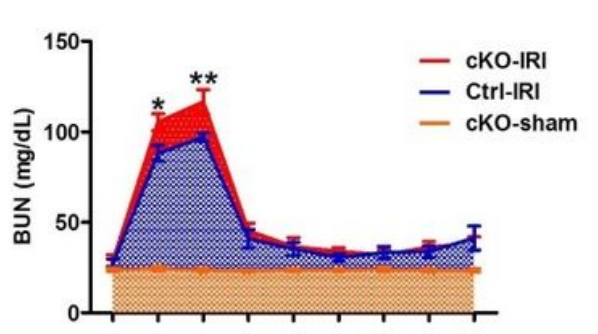

C

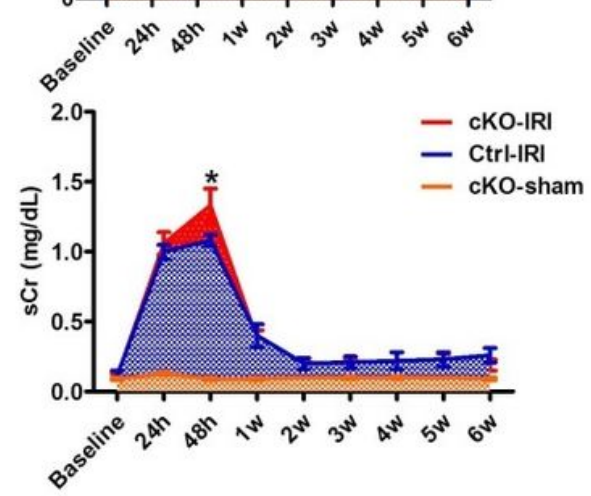

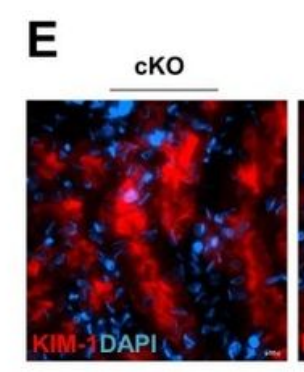

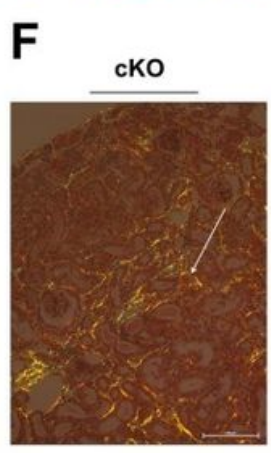

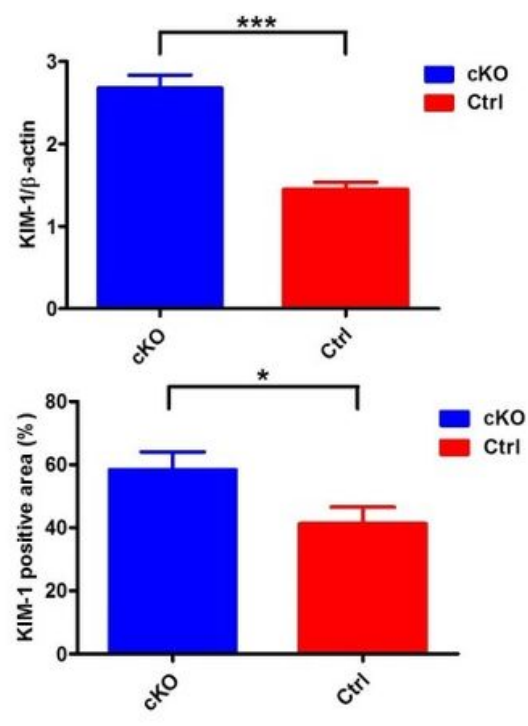
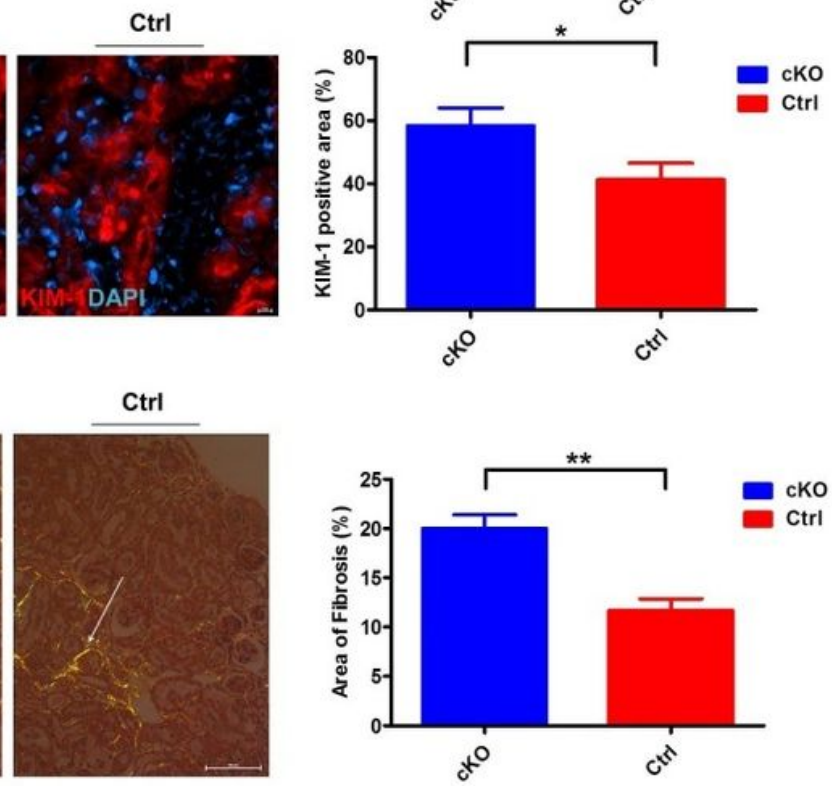

\section{Figure 2}

Functional and histological change of kidney from IRI (A) Ideograph of mouse renal IRI model. (B-C) Recovery patterns of BUN and SCr from IRI. (D-E) KIM-1 expressions in renal cortex (2 days after ischemia), detecting by western blot and immunofluorescence staining. (F) Severity of tubulointerstitial fibrosis (42 days after ischemia), detecting by picrosirius red staining. Abbreviations BUN blood urea nitrogen, cKO conditional knockout, Ctrl control, KIM-1 kidney injury molecule-1, SAC sacrifice, sCr serum creatinine, IRI ischemia-reperfusion injury * meant $p<0.05$, ** meant $p<0.01$, *** meant $p<0.001$, were assumed as statistically significant. Scale bar: $10 \square \mathrm{m}(\mathrm{E}), 100 \mathrm{\square m}(\mathrm{F})$ 


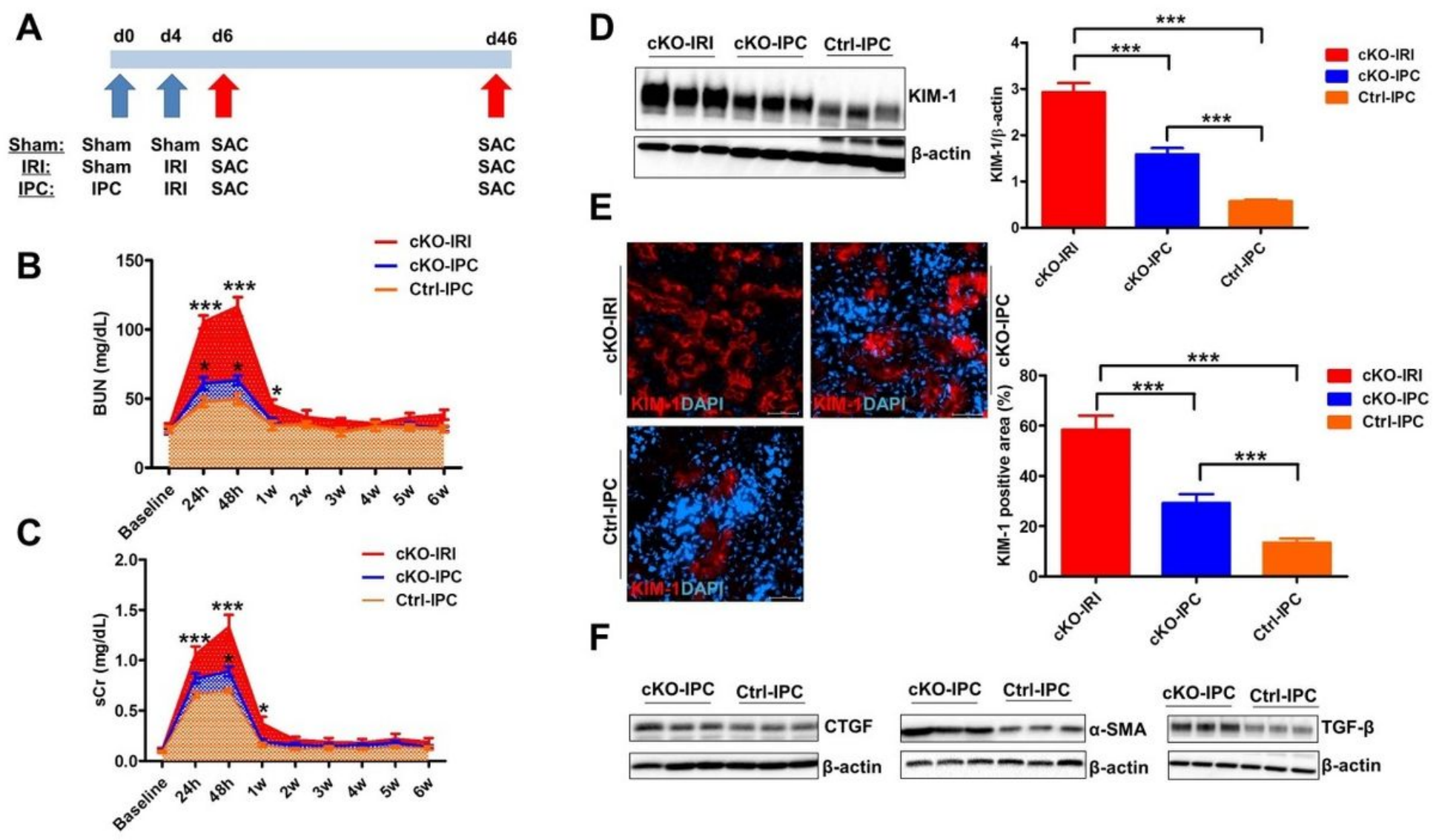

\section{Figure 3}

Acute phase assessment after renal IPC (A) Ideograph of IPC strategy. (B-C) Recovery patterns of BUN and $\mathrm{sCr}$ after IPC. (D-E) KIM-1 expressions in renal cortex (2 days after the index ischemia), detecting by western blot and immunofluorescence staining. (F) Renal protein levels of the profibrotic and fibrotic marker, including CTGF, a-SMA and TGF- $\beta$, etc. (2 days after the index ischemia), detecting by western blot. Abbreviations a-SMA alpha smooth muscle actin, BUN blood urea nitrogen, cKO conditional knockout, CTGF connective tissue growth factor, Ctrl control, IPC ischemic preconditioning, IRI ischemiareperfusion injury, KIM-1 kidney injury molecule-1, SAC sacrifice, $\mathrm{s} C$ r serum creatinine, TGF- $\beta$ transforming growth factor $\beta$ * meant $p<0.05$, $* \star \star$ meant $p<0.001$, were assumed as statistically significant. Scale bar: $50 \square \mathrm{m}(\mathrm{E})$ 


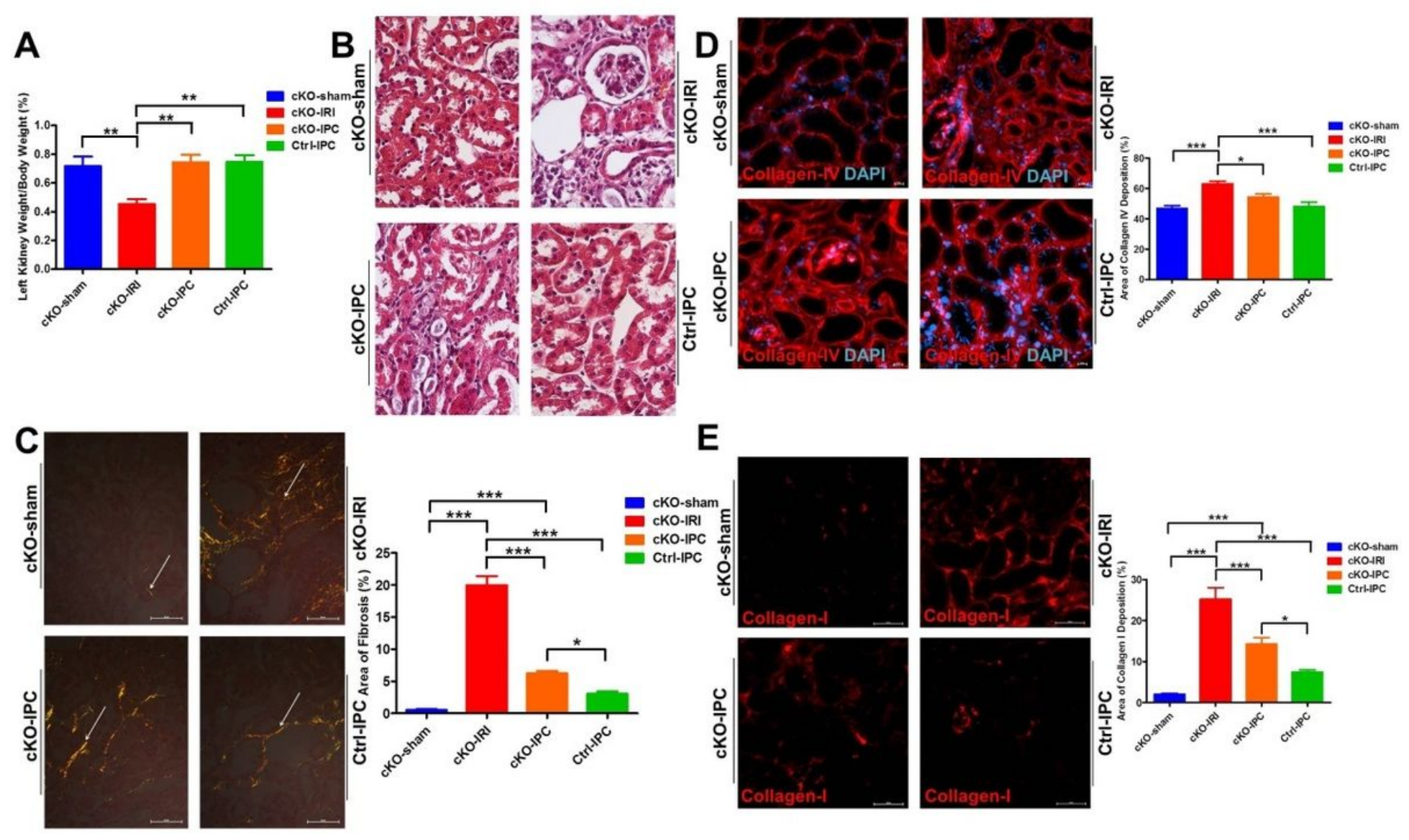

Figure 4

Chronic phase assessment after renal IPC (A) Severity of kidney atrophy after IPC, represented by left kidney weight / body weight (\%). (B) Histological change of kidney after IPC, detecting by H\&E staining. (C-E) Severity of tubulointerstitial fibrosis, detecting by picrosirius red staining and immunofluorescence staining of collagen IV and I. The time point assessment was 42 days after the index ischemic injury. Abbreviations cKO conditional knockout, Ctrl control, IPC ischemic preconditioning, IRI ischemiareperfusion injury * meant $p<0.05$, ** meant $p<0.01$, *** meant $p<0.001$, were assumed as statistically significant. Scale bar: $10 \square m(B, D), 100 \square m(C, E)$

\section{Supplementary Files}

This is a list of supplementary files associated with this preprint. Click to download.

- NC3RsARRIVEGuidelinesChecklistfillable.pdf 